\title{
The position angle of the Galactic bulge
}

\author{
A. Vallenari ${ }^{1}$, S. Ragaini ${ }^{1}$ and G. Bertelli ${ }^{1}$ \\ ${ }^{1}$ INAF, Padova Astronomical Observatory, vicolo Osservatorio 5, I-35122, Padova, Italy \\ email: antonella.vallenari@oapd.inaf.it, silvia.ragaini@oapd.inaf.it, \\ gianpaolo.bertelli@oapd.inaf.it
}

\begin{abstract}
We present a study of the structure of the Galactic Bulge in the region $-6^{\circ}<b<$ $3^{\circ},-17^{\circ}<l<17^{\circ}$ using 2MASS archive data. More than 100 fields are used. We make use of the red clump method to derive the distance of the Bulge in the studied regions. We derive a position angle of the Galactic Bulge going from $42^{\circ} \pm 11$ to $35^{\circ} \pm 10$ depending on the adopted bulge mass distribution.
\end{abstract}

Keywords. Galaxy: structure, Galaxy: stellar content, stars: mass function

\section{Introduction}

The position angle of the inner $\left(1<10^{\circ}\right)$ bulge/bar with the direction Sun-Galactic center is still very uncertain. While kinematics determinations based on $\mathrm{OH} / \mathrm{IR}$ stars suggest a large angle of about $40^{\circ}$ (Sevenster et al. 1999), photometry determinations still give contrasting results, going from $40^{\circ}$ (van Loon et al. 2003) down to about $12^{\circ}$ (Binney et al. 1997). In addition, it is still matter of discussion whether the Galactic bulge and the Galactic bar are two separate structures, having different position angles and being located in the inner and external regions, respectively. Suggestions in that sense have been advanced in literature (López-Corredoira et al. 2007; Mahoney 2007). If the bulge is formed by secular evolution of the bar, then these structures are expected to have the same position angle.

\section{Results}

As a part of a project aimed to model the Galaxy structure, in this paper we discuss the position angle of the Bulge in the region $-17^{\circ}<l<17^{\circ}$ and $-6^{\circ}<b<3^{\circ}$ using 100 stellar fields of 2 MASS survey. We make use of the red clump stars as tracers of the bulge population. We derive the theoretical clump absolute magnitude and color from the simulations done by means of the Padova isochrones assuming an age of 12-10 Gyr, $\mathrm{Z}=0.008-0.02$. We obtain $\mathrm{M}_{K}=-1.55,\left(H-K_{0}\right)=0.064$. We make use of the extinctionfree magnitude defined as: $K_{H-K} \equiv K_{S}-\frac{A_{K_{S}}}{E\left(H-K_{S}\right)}\left[\left(H-K_{S}\right)-\left(H-K_{S}\right)_{0}\right]$ where the used reddening law is $A_{K_{S}} / E\left(H-K_{S}\right)=1.4$. In this way, $K_{H-K}$ is independent from the extinction if the extinction law $A_{K_{S}} / E\left(H-K_{S}\right)$ is independent from the location. The red clump method, as often applied in literature, relies on the assumption that the maximum of the star density in the red clump is located on the major axis of the bulge. In general, this is not strictly true, since the maximum of the density is located at the tangential point of the line of sight with the ellipsoids describing the bulge isodensity contours. The location of the maximum of the density can be far away from the major axis as much as $1 \mathrm{Kpc}$, depending on the bulge intrinsic shape.To derive the maxima of the star density at each value of $(1, b)$ we make use of the bulge density distributions included in the Padova Galaxy Model (Vallenari \& Ortolani 2001), taken from 


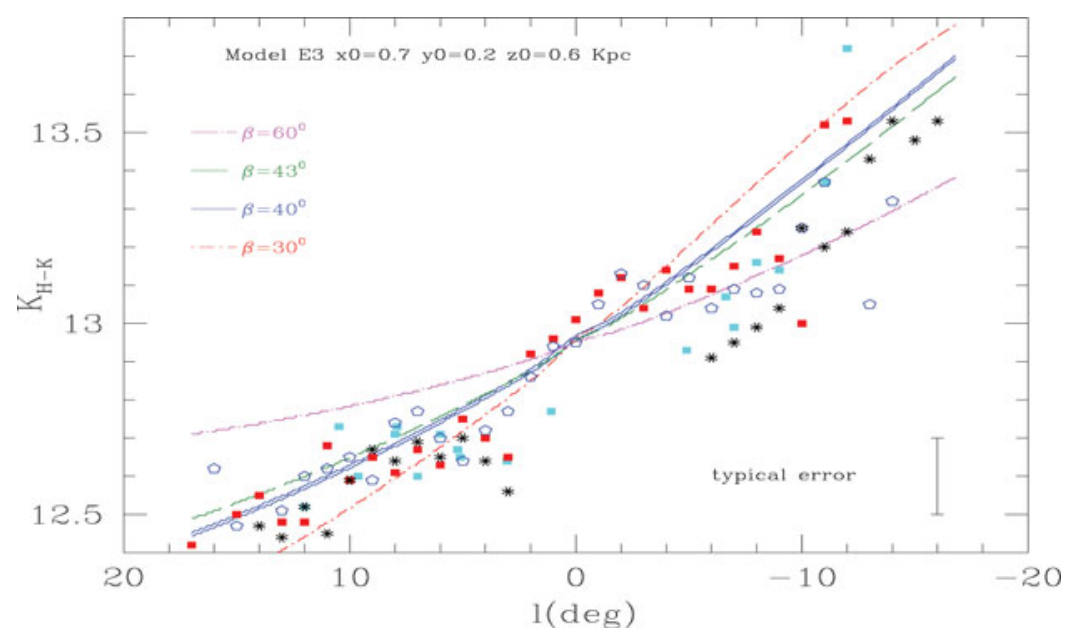

Figure 1. Observational reddening free $\mathrm{K}_{H-K}$ magnitude of the red clump peak in the studied regions as a function of the longitude 1 . Squares indicate the data at $b=-3^{\circ}$ and $b=-6^{\circ}$, penthagons show the data at $\mathrm{b}=-5^{\circ}$, stars present the data at $\mathrm{b}=+3^{\circ}$. The lines give the theoretical magnitudes of the red clump at changing values of the position angle $\beta$ for the model E3 when the distance Sun-GC is assumed to be $7.9 \mathrm{Kpc}$

Dwek et al. (1995) distributions, namely their G2 "boxy" Gaussian models, their exponential-type boxy model E3, the modified Bahcall distribution G3. Preliminary results show that the data are in agreement with a position angle $\beta=42^{\circ} \pm 11$ when the E3 model is used. The result does not change significantly when the G2 model is used, but the fit is definitively worse, even if the semi-axis dimensions $\left(x_{o}, y_{o}, z_{o}\right)$ change. With these two boxy models, a poor fit is obtained when $\beta<30^{\circ}$. If the G3 model is assumed, then the position angle is $\beta=35 \pm 10^{\circ}$. Fig. 1 presents the observational red clump magnitudes compared with the expected values for the E3 model. Using as distance indicator the magnitude of the red giant clump, Nishiyama et al. (2005) found evidence of a structure in the inner $l=|4|^{\circ}$ at very low Galactic latitude $\mathrm{b} \sim+1$. This feature has been interpreted as a distinct bar in the inner Galaxy. Our analysis suggests that this bar is not visible at $|b|>3^{\circ}$ and is well confined on the galactic plane (Vallenari, Ragaini \& Bertelli 2007).

\section{References}

Binney, J., Gerhard, O., \& Spergel, D. 1997, MNRAS 288, 365

Dwek, E., Arendt, R. G., Hauser, M. G., et al. 1995, ApJ 445, 716

López-Corredoira, M., Cabrera-Lavers, A., Mahoney, T. J., Hammersley, P. L., Garzón, F., \& González-Fernández, C. 2007, AJ 133, 154

Mahoney, T. J. 2007, this meeting

Nishiyama, S., Nagata, T., Baba, D., et al. 2005, ApJ 621, 105

Sevenster, M., Saha, P., Valls-Gabaud, D., \& Fux, R. 1999, MNRAS 307, 584

Vallenari, A. \& Ortolani, S. 2001, A\&SA 380, 35

Vallenari, A., Ragaini, S., \& Bertelli, G. 2007 A\&A, submitted

van Loon, J. T., Gilmore, G. F., Omont, et al. 2003, MNRAS 338, 857 


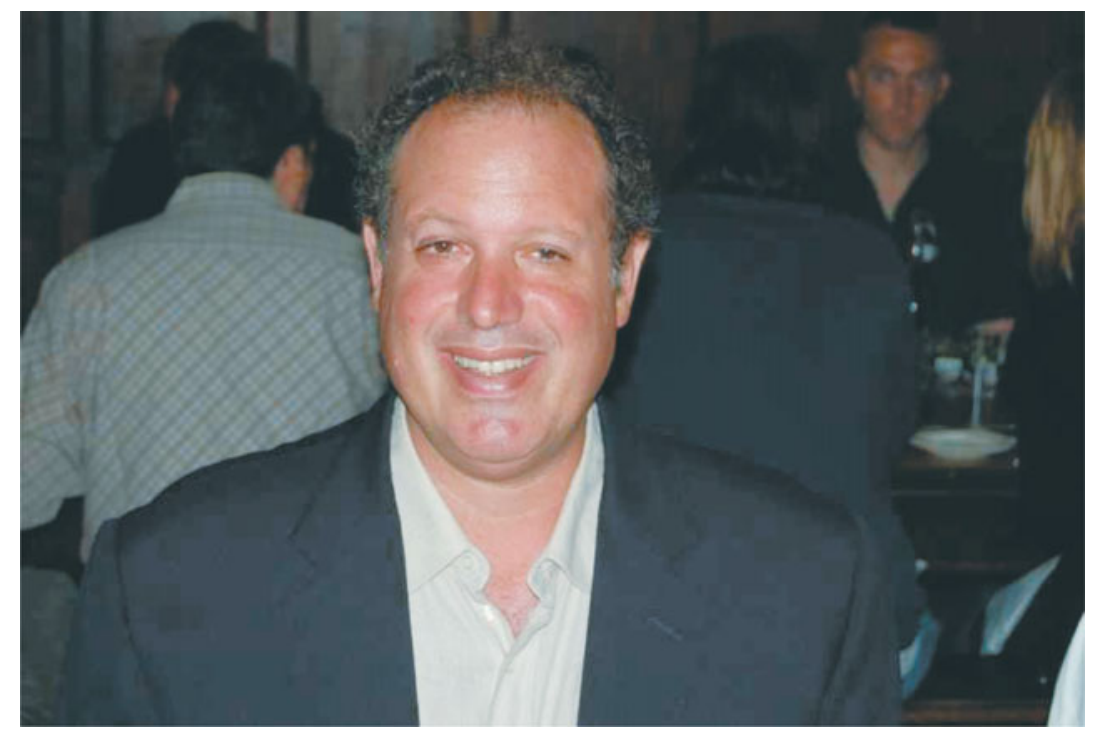

Figure 2. Michael Rich during the symposium dinner.

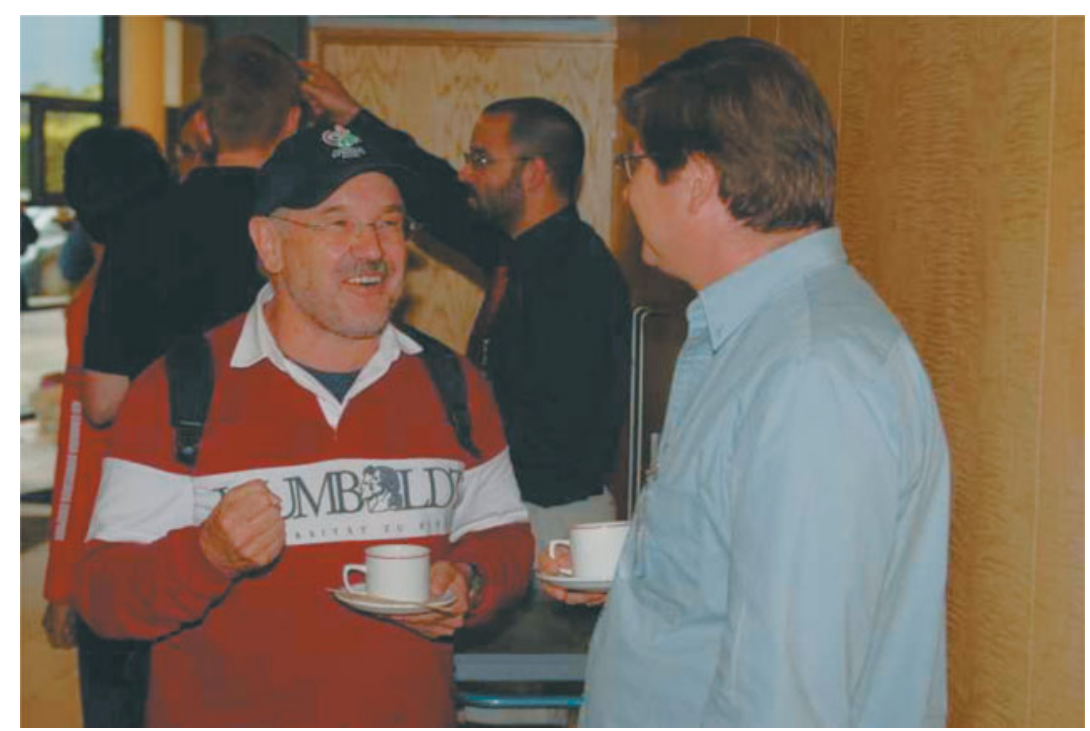

Figure 3. Hans Zinnecker and SOC member Dante Minniti discussing during a coffee break. George Bendo is visible in the centre background. 


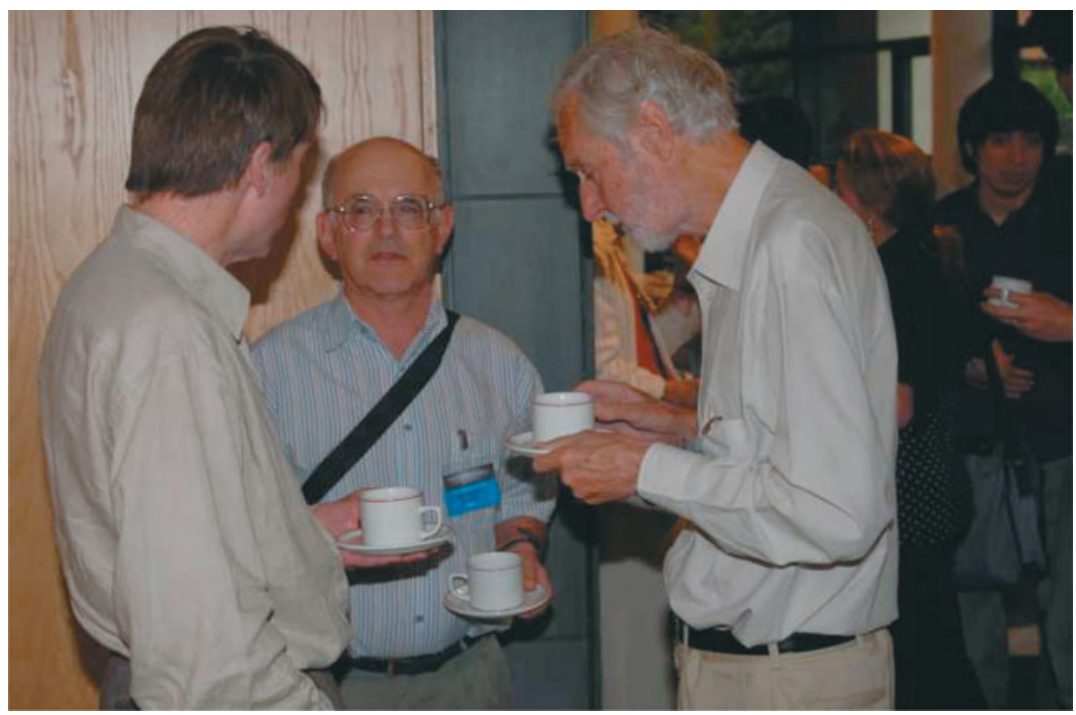

Figure 4. Mark Cropper, SOC member Ken Freeman and Michael Feast discussing during a coffee break.

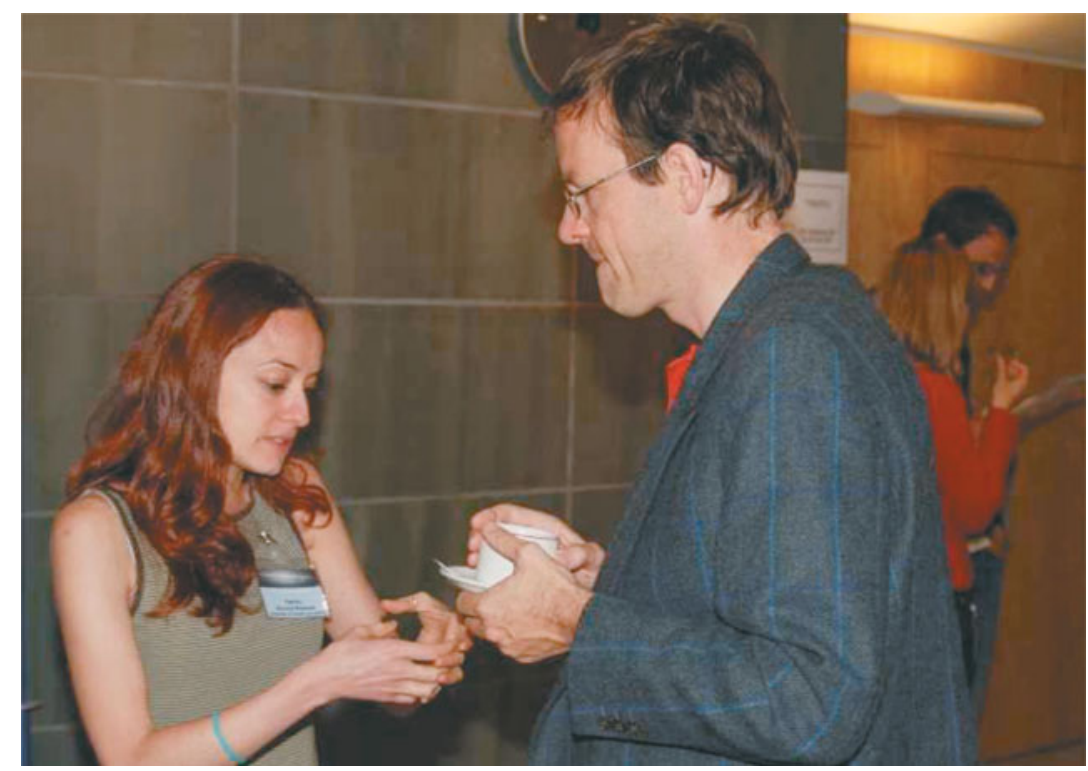

Figure 5. Patricia Sánchez-Blázquez and Reynier Peletier discussing during a coffee break. 


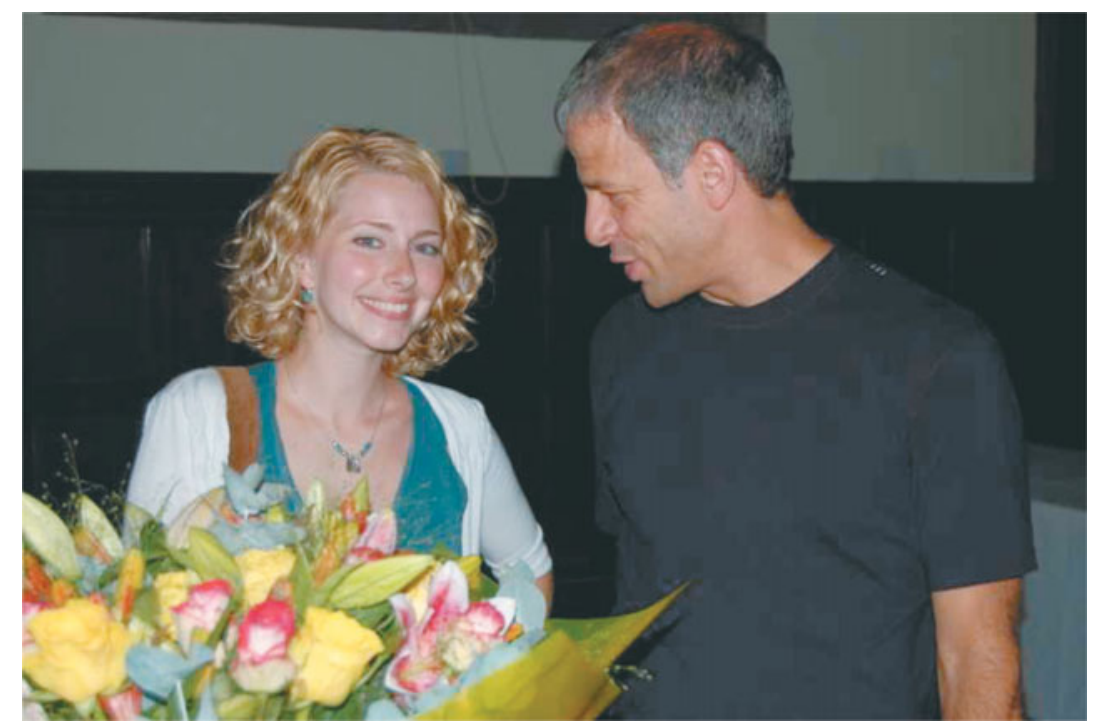

Figure 6. LOC member Millie Maier and Stéphane Courteau discussing after the symposium dinner.

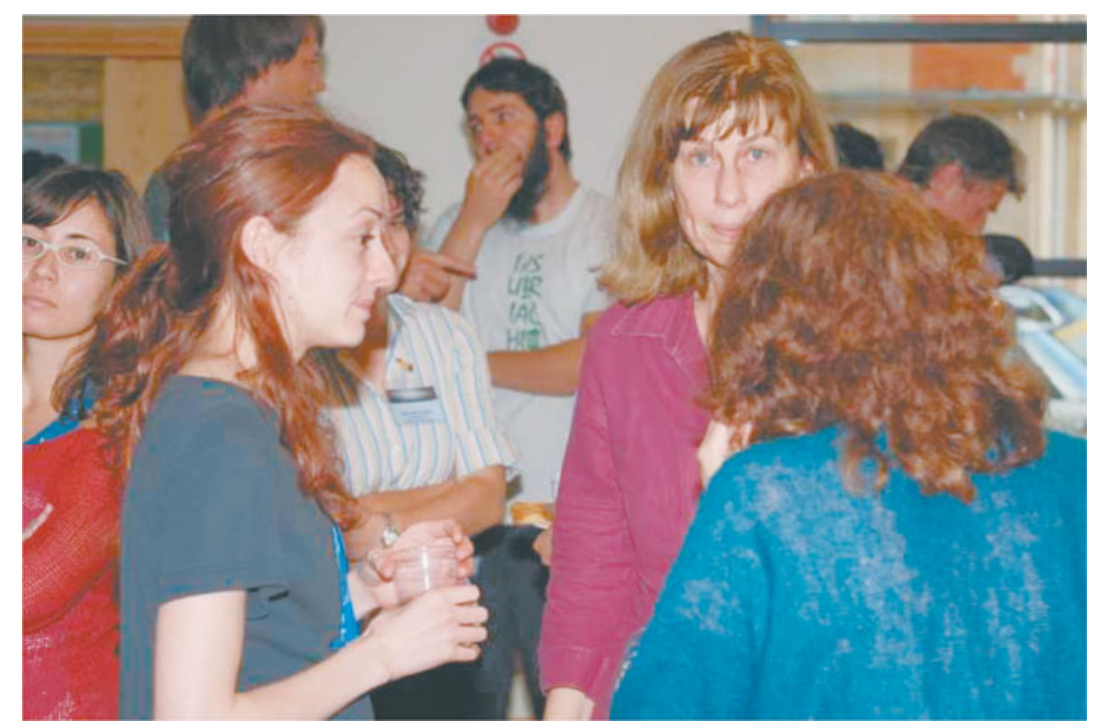

Figure 7. Patricia Sánchez-Blázquez, Beatriz Barbuy and Anne Sansom discussing during a coffee break. Lilian Domínguez Palmero (left), Marc Freitag, Genevieve Graves, Alberto Sesana (centre) and SOC member Matthias Steinmetz (right) are visible in the background. 


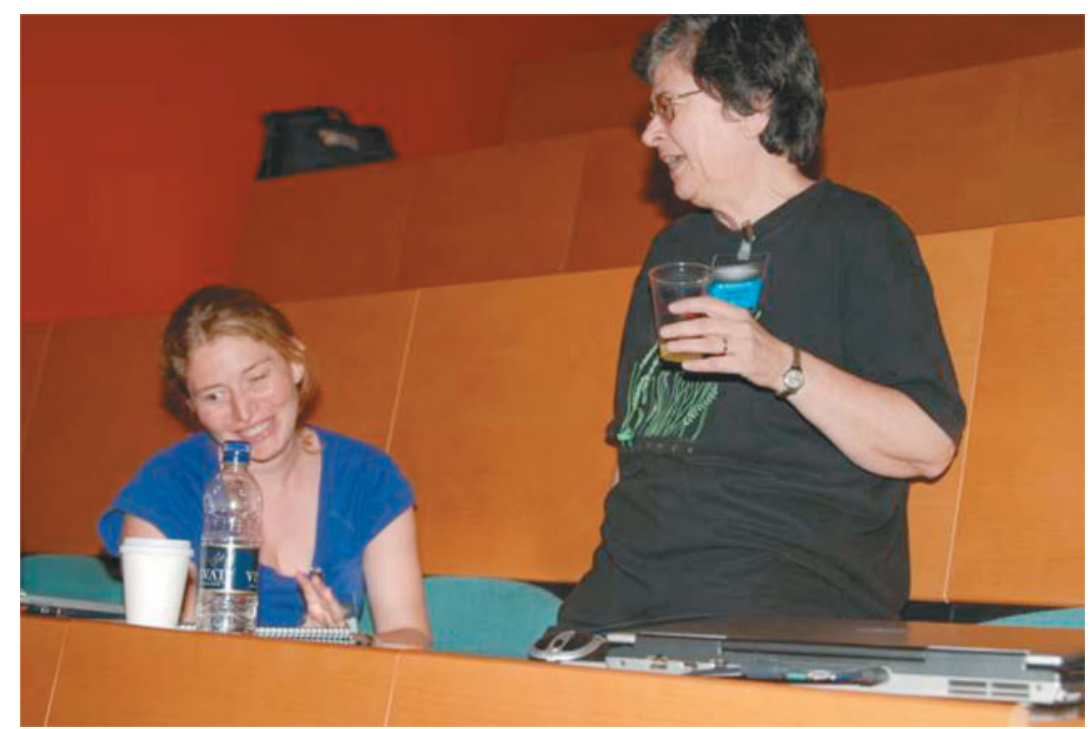

Figure 8. Inma Martinez-Valpuesta and SOC member Lia Athanassoula discussing in the lecture theatre.

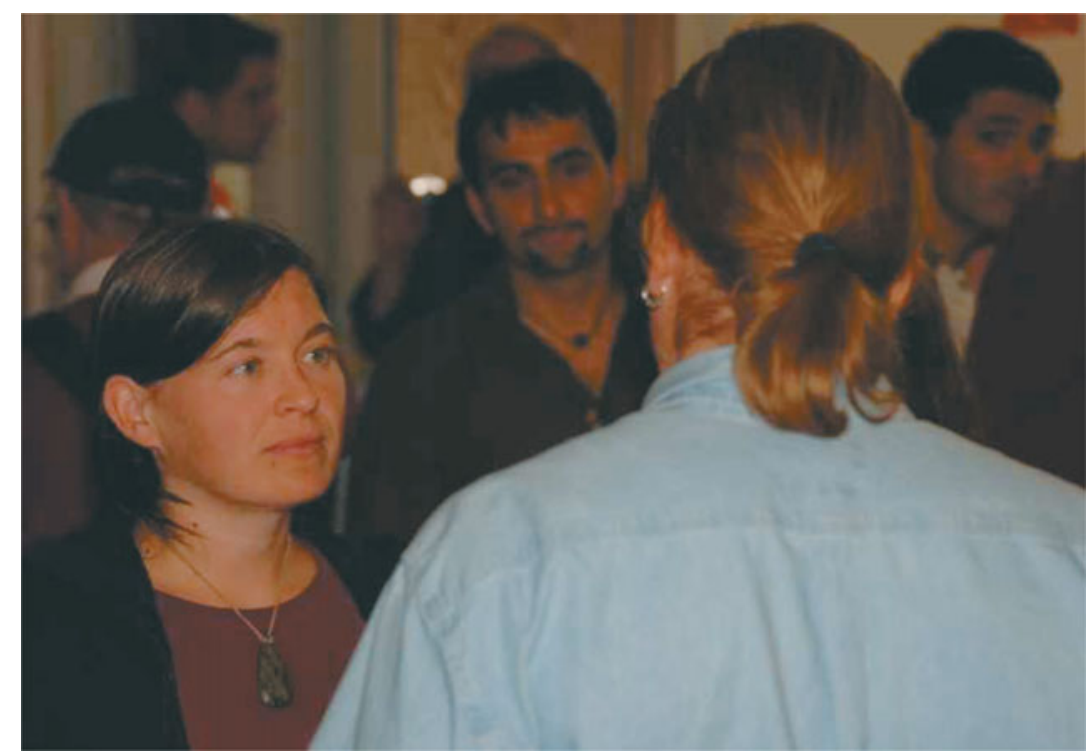

Figure 9. Nicola Bennert and Dimitri Gadotti discussing during a coffee break. LOC member Marc Sarzi (centre) and Sadegh Khochfar (right) are visible in the background. 